\title{
Technical note: Evaluation of an ear-attached movement sensor to record cow feeding behavior and activity
}

\author{
J. P. Bikker, ${ }^{\star 1}$ H. van Laar, ${ }^{\star} †$ P. Rump,‡ J. Doorenbos,† K. van Meurs, $\neq$ G. M. Griffioen,‡ and J. Dijkstra* \\ ${ }^{*}$ Animal Nutrition Group, Wageningen University, PO Box 338, 6700 AH Wageningen, the Netherlands \\ †Nutreco R\&D, PO Box 220, 5830 AE Boxmeer, the Netherlands \\ $\ddagger$ Agis Automatisering, PO Box 46, 3480 DA, Harmelen, the Netherlands
}

\section{ABSTRACT}

The ability to monitor dairy cow feeding behavior and activity could improve dairy herd management. A 3-dimensional accelerometer (SensOor; Agis Automatisering BV, Harmelen, the Netherlands) has been developed that can be attached to ear identification tags. Based on the principle that behavior can be identified by ear movements, a proprietary model classifies sensor data as "ruminating," "eating," "resting," or "active." The objective of the study was to evaluate this sensor on accuracy and precision. First, a pilot evaluation of agreement between 2 independent observers, recording behavior from 3 cows for a period of approximately 9 $\mathrm{h}$ each, was performed. Second, to evaluate the sensor, the behavior of 15 cows was monitored both visually (VIS) and with the sensor (SENS), with approximately $20 \mathrm{~h}$ of registration per cow, evenly distributed over a 24-h period, excluding milking. Cows were chosen from groups of animals in different lactation stages and parities. Each minute of SENS and VIS data was classified into 1 of 9 categories ( 8 behaviors and 1 transition behavior) and summarized into 4 behavioral groups, namely ruminating, eating, resting, or active, which were analyzed by calculating kappa $(\kappa)$ values. For the pilot evaluation, a high level of agreement between observers was obtained, with $\kappa$ values of $\geq 0.96$ for all behavioral categories, indicating that visual observation provides a good standard. For the second trial, relationships between SENS and VIS were studied by $\kappa$ values on a minute basis and Pearson correlation and concordance correlation coefficient analysis on behavior expressed as percentage of total registration time. Times spent ruminating, eating, resting, and active were 42.6, 15.9, 31.6, and $9.9 \%$ (SENS) respectively, and 42.1, 13.0, 30.0, and $14.9 \%$ (VIS), respectively. Overall $\kappa$ for the comparison of SENS and VIS was substantial (0.78), with $\kappa$ values of $0.85,0.77,0.86$, and 0.47 for "ruminating," "eating," "resting," and "active," respectively. Pearson correla-

Received October 3, 2013.

Accepted February 5, 2014

${ }^{1}$ Corresponding author: jasper.bikker@gmail.com tion and concordance correlation coefficients between SENS and VIS for "ruminating," "eating," "resting," and "active" were $0.93,0.88,0.98$, and 0.73 , and 0.93 , $0.75,0.97$, and 0.35 , respectively. In conclusion, the results provide strong evidence that the present ear sensor technology can be used to monitor ruminating and resting behavior of freestall-housed dairy cattle. Our results also suggest that this technology shows promise for monitoring eating behavior, whereas more work is needed to determine its suitability to monitor activity of dairy cattle.

Key words: sensor technology, dairy cow behavior, ruminating

\section{Technical Note}

In many areas of the world the number of cows per dairy farm has increased and, as a consequence, the time farmers spend on individual cows has been reduced. This trend, driven by reducing workload and increasing efficiency and profitability, has resulted in a rapid increase in the interest of farmers and industry for precision dairy farming technology. Precision dairy farming technology aims to support decision making and to improve farm productivity and profitability (van Asseldonk et al., 1999).

Daily ruminating, eating, and activity patterns are widely considered as indicators closely related to health events and productivity for individual cows. For research, easy measurement of these parameters may help understanding of nutritional physiology. In dairy farming, to minimize loss of milk yield and of health costs, it is essential to observe physiological and behavioral changes or abnormalities as early as possible. Therefore, automatic monitoring of feeding behavior and activity could provide scientists and farmers a research tool and an early warning tool, respectively. Already several technologies have been developed to monitor activity (e.g., Walker et al., 1996; Roelofs et al., 2005), feeding (Beauchemin et al., 1989; Rutter et al., 1997; Kononoff et al., 2002), and (or) ruminating (Schirmann et al., 2009; Burfeind et al., 2011; Gold- 
hawk et al., 2013) behavior. However, these systems either can measure only one type of behavior or are less suitable for practical use.

Recently, an electronic behavior sensor, the CowManager SensOor system (Agis Automatisering BV, Harmelen, the Netherlands) was introduced. This system enables real-time quantification of (multi-point) ear temperature, ruminating and eating behavior, and activity of dairy cows. A scientific evaluation on the accuracy and precision of the behavioral model has not been carried out. Therefore, the overall objective of this study was to determine the agreement between the classifications of behavior into categories based on the sensor against direct visual observations.

The study was conducted between February and March 2013 on the experimental dairy farm de Kempenshof (Nutreco Ruminant Research Centre, Boxmeer, the Netherlands), in compliance with the Dutch law on experimental animals. Cows were housed together in a freestall barn with a slatted floor. Lactating cows were milked twice daily with a $12-\mathrm{h}$ interval. Two evaluations were performed. First, the agreement of classification of direct visual observation of 2 independent human observers was evaluated. Second, agreement of classification of the sensor versus visual observation was evaluated.

The sensor in the CowManager SensOor system is a molded microchip that has to be clicked into an adapted ear identification tag (Supertag; Dalton ID Ltd., Oxfordshire, UK). A 3-dimensional accelerometer continuously registers the movements of the cow's ear. Data are sent through a wireless connection, via routers and coordinators, to a computer. The data logger allows data storage for a maximum of $48 \mathrm{~h}$ after the last communication. Raw data are continuously collected and each minute is classified as 1 of 4 behavioral categories, namely "ruminating," "eating," "resting," and "active," with a proprietary model, which are subsequently expressed as percentage of behavior per hour as well as per day and are available through a web-based application.

For the first experiment, 2 observers with extensive previous experience were trained to classify visual observations of cow behavior (Table 1). Before the experiment, both observers discussed the definition of the different behaviors until agreement was reached. The observers independently recorded cow behavior from 3 lactating cows, every minute during a period of approximately $9 \mathrm{~h}$. During this period, the observers did not communicate with each other and were unable to view each other's scoring. The observers were positioned outside the area of the stable where the 3 cows were housed, with an unobstructed view on the individual cows.
For the second experiment, the required number of cows for the evaluation of sensor versus human observer was based on a statistical power analyses for correlation as described by Friedman (1982). To obtain 0.90 power with an $\alpha$ of 0.05 and an assumed effect size of 0.70 (= correlation coefficient as a measure of effect size), a minimum number of 13 cows was required. In the present trial, 15 Holstein-Friesian cows from different parity and lactation stage combinations in the same free stable were selected to be used, resulting in 5 cow groups of 3 cows initially. Cow groups were characterized as below 50 DIM $(33.5 \pm 3.94 \mathrm{~kg}$ of milk/d; mean $\pm \mathrm{SD})$ or above 250 DIM $(24.6 \pm 7.27 \mathrm{~kg}$ of milk/d), and first parity $(29.2 \pm 9.56 \mathrm{~kg}$ of milk/d) versus third parity and higher $(28.0 \pm 4.41 \mathrm{~kg}$ of milk/d), leading to 4 groups. A fifth group comprised dry cows. One cow (parity $3 ; \geq 250$ DIM) was removed from analysis, because of strongly altered behavior, most probably due to problems in another experiment. One extra dry cow was observed, making the final selection again 15 Holstein-Friesian cows.

Cows were part of different experiments and were fed different diets. Seven cows were fed a partial mixed ration (PMR) comprising $42.5 \%$ grass silage, $45.9 \%$ maize silage, and $11.6 \%$ hay on a DM basis (the PMR contained $41.5 \% \mathrm{DM})$. Dependent on production level, the PMR was supplemented with concentrate fed in automatic feeders. Four cows were fed a TMR comprising $23.2 \%$ grass silage, $25.4 \%$ maize silage, $11.6 \%$ hay, and $39.8 \%$ concentrate on a DM basis (the TMR contained $54.7 \%$ DM). The 4 dry cows were fed a PMR as well, comprising $12.9 \%$ grass silage, $46.8 \%$ maize silage, $6.9 \%$ hay, and $33.4 \%$ straw (the PMR contained $47.7 \%$ DM), supplemented with $4 \mathrm{~kg} / \mathrm{d}$ of a close-up concentrate in an automatic feeder. The PMR and TMR were fed ad libitum in roughage intake control (RIC) feeders, which were filled once daily at $1000 \mathrm{~h}$.

For a period of $4 \mathrm{~d}$, the behavior of the 15 cows was monitored with sensor and visual observation in blocks of varying hours (1-7), with approximately $20 \mathrm{~h}$ of registration per cow evenly distributed over a 24 -h period, excluding milking $(2 \times 2 \mathrm{~h})$. For visual observation, the 15 cows were divided over the same 2 observers who carried out the human versus human evaluation. Before every observation the session time was calibrated by synchronizing the windows system clock with that at http://time.windows.com. For human observations, the behaviors of a cow were classified by minute, in agreement with the ethogram as presented in Table 1 and were processed and condensed to 4 categories, namely "ruminating," "eating," "resting," and "active," with transitional minutes removed. The same 4 condensed categories (see Table 1) were used to classify the sensor data using a proprietary model. The condensed behav- 
ioral category resting was composed of initial categories "lying resting" and "standing inactive." To minimize the risk of influence of sensor position in the ear on the recordings, all sensors were placed $6.6 \pm 1.5 \mathrm{~cm}$ out of the concha and centered between the 2 typical major veins across the cow's ear.

Various approaches were used to evaluate the correspondence between individual observers and between visual observations and sensor recordings, including measures of interrater agreement (kappa statistic), paired $t$-test, and concordance correlation analysis. For analysis in the first experiment, the kappa $(\kappa)$, standard error of $\kappa$, and maximum kappa $\left(\kappa_{M a x}\right)$ statistics (Cohen, 1960) were calculated for individual behavioral categories from Table 1 (behavior versus "other" behavior) and for an overall comparison. The $\kappa$ value provides a measure for minute-by-minute agreement between observers, corrected for agreement that would be expected by chance. The $\kappa_{M a x}$ indicates the maximum value of $\kappa$ that can be achieved when a difference exists between observers (or observer and sensor) in the number of minutes scored for each behavioral category. The $\kappa_{\text {Max }}$ helps to interpret $\kappa$ values obtained, with low $\kappa_{M a x}$ values indicating that the criteria to distinguish categories are not clear (Cohen, 1960). To enable subsequent comparison with the sensor of the second experiment, the 8 behavioral categories were condensed into 4 categories, namely ruminating, eating, resting, and active, with transitional minutes removed. For these condensed categories, $\kappa$, standard error of $\kappa$, and $\kappa_{\text {Max }}$ were calculated for both experiments. The $\kappa$ values were judged according to criteria of Landis and Koch (1977), namely poor $(<0.00)$, slight $(0.00-0.20)$, fair (0.21-0.40), moderate $(0.41-0.60)$, substantial (0.61-0.80), and almost perfect (0.81-1.00).

Additionally, for the second experiment, data were analyzed per cow as percentage of behavior per total time registered, using SAS (version 9.2; SAS Institute Inc., Cary, NC). Agreement between sensor and human observation was evaluated using a 2 -sided paired $t$-test. Relationships between sensor and visual observations were further studied by performing a Pearson correlation. Subsequently a concordance correlation coefficient (CCC; Lin, 1989) analysis was carried out with the equations presented by Ellis et al. (2010). The CCC analysis provides an integrated measure of correlation as well as accuracy and precision. A difference in standard deviation between sensor and visual observation is indicated by the scale shift and under- or overprediction is indicated by a location shift. A negative value for the location shift indicates an overprediction, whereas a positive value indicates underprediction. Pearson correlation and $\mathrm{CCC}$ were judged according to the criteria of Hinkle et al. (2003), namely negligible (0.00-0.30), low 
Table 2. Classification of behavior within the same minute for each cow, expressed as the number of minutes of the registered behavior for 2 (A vs. B) human observers, with associated kappa $(\kappa) \pm \mathrm{SE}$ and maximum kappa $\left(\kappa_{\text {Max }}\right)$ values

\begin{tabular}{|c|c|c|c|c|c|c|}
\hline Item & $\begin{array}{c}\text { Ruminating } \\
\text { (A) }\end{array}$ & $\begin{array}{l}\text { Eating } \\
\text { (A) }\end{array}$ & $\begin{array}{l}\text { Resting } \\
\text { (A) }\end{array}$ & $\begin{array}{l}\text { Active } \\
\text { (A) }\end{array}$ & $\begin{array}{c}\text { Total } \\
\text { observer B }\end{array}$ & $\kappa\left(\kappa_{M a x}\right)^{1}$ \\
\hline \multicolumn{7}{|c|}{ Behavior classification } \\
\hline Resting (B) & & & 320 & 4 & 324 & $0.97 \pm 0.07(0.99)$ \\
\hline Active (B) & & & 11 & 273 & 284 & $0.96 \pm 0.08$ \\
\hline Total observer A & 419 & 367 & 331 & 280 & 1,397 & $0.98 \pm 0.04(0.99)$ \\
\hline
\end{tabular}

${ }^{1}$ Kappa $(\kappa)$ and $\kappa_{\text {Max }}$ values as calculated based on Cohen (1960). The $\kappa$ value provides a measure for minute-by-minute agreement between observers, corrected for agreement that would be expected by chance. The $\kappa_{\text {Max }}$ indicates the maximum value of $\kappa$ that can be achieved when a difference exists between observers (or observer and sensor) in the number of minutes scored for each behavioral category.

(0.30-0.50), moderate (0.50-0.70), high (0.70-0.90), and very high $(0.90-1.00)$.

In the first experiment, the 3 cows were observed for a total of $521 \mathrm{~min}$, resulting in a pooled set of observations of 1,563 min in total. Of those observations, 142 min were classified as transitional behavior by both observers and an additional 24 min were classified as transitional behavior by one of the observers, resulting in 1,397 useable minute observations. For the initial behavioral categories (Table 1) agreement based on $\kappa$ was almost perfect as judged by Landis and Koch (1977), except for the categories standing active, standing medium active, and standing high active, for which agreement was substantial or moderate. For the latter categories $\kappa_{\text {Max }}$ was relatively low, indicating that the criteria to distinguish these categories were not clear. For the condensed behavioral categories (Table 2 ), agreement based on $\kappa$ was high (almost perfect) for ruminating and eating with $\kappa$ values of 1.00 each. Agreement was slightly lower (still almost perfect) for resting (0.97) and active (0.96).

For the second experiment, the evaluation of sensor versus visual observation, $1,105 \pm 19$ (mean $\pm \mathrm{SD}$ ) min of observation per cow were used. Of the potential $1,200 \mathrm{~min}$ of observations/cow, $13 \pm 12 \mathrm{~min} /$ cow were not scored and $83 \pm 16 \mathrm{~min} / \mathrm{cow}$ were scored as transitional minute. The sensor performed well without any data loss.

Table 3 presents the absolute comparison between sensor and visual observation on time spent for each behavior category as percentage of total recorded time. Table 4 presents the $\kappa$ and $\kappa_{M a x}$ values, Pearson correlation, and CCC analysis for the relationship between sensor and visual observations of behavior.

Proportion of time ruminating did not differ $(P=$ 0.49 ) between sensor and visual observation. For ruminating, the minute-by-minute agreement was almost perfect ( $\kappa$ value of 0.85 ). For agreement on percentage of time ruminating, the Pearson correlation and CCC between sensor and visual observation were both very high. The minor differences in absolute levels and stan- dard deviation between sensor and human estimated ruminating times were confirmed by a location and scale shift close to 0 and to 1 , respectively, leading to a CCC equal to Pearson correlation.

Percentage of time eating as recorded by the sensor was $2.9 \%$ units higher $(P=0.001)$ than by visual observation. For eating, minute-by-minute agreement was substantial, with a $\kappa$ value of 0.77 . For percentage of time agreement the Pearson correlation and CCC between sensor and visual observation were both high. The difference in absolute level of percentage of time eating was reflected in a location shift of -0.58 , which is the main reason for the CCC being lower than the Pearson correlation. However, the standard deviation of sensor and human estimated eating times was similar, with a scale shift close to 1 .

The percentage of time resting as recorded by the sensor was $1.6 \%$ units higher $(P=0.024)$ than for visual observation. For resting, minute-by-minute agreement was almost perfect ( $\kappa$ value of 0.86 ). For percentage of time agreement, the Pearson correlation and CCC between sensor and visual observation were both very high. Although the difference of $1.6 \%$ units was statistically significant, the impact on the relationship between sensor and human estimated resting times was small, as location and scale shift were close to 0 and to 1 , respectively, leading to a slightly lower CCC (0.01 lower) with respect to the Pearson correlation.

The percentage of time active as recorded by the sensor was $5.0 \%$ units lower $(P<0.001)$ than for visual observation. For activity, minute-by-minute agreement

Table 3. Percentage of time performing specific behavior of total recorded time $\pm \mathrm{SD}$ for sensor versus visual observation and $P$-values for the 2 -sided paired $t$-test

\begin{tabular}{lccc}
\hline Behavior & Sensor & \multicolumn{1}{c}{ Visual } & $P$-value \\
\hline Ruminating & $42.6 \pm 6.81$ & $42.1 \pm 6.94$ & 0.49 \\
Eating & $15.9 \pm 5.02$ & $13.0 \pm 4.94$ & 0.001 \\
Resting & $31.6 \pm 10.96$ & $30.0 \pm 11.59$ & 0.024 \\
Active & $9.9 \pm 2.78$ & $14.9 \pm 5.71$ & $<0.001$ \\
\hline
\end{tabular}


Table 4. Pearson correlation coefficient $(\mathrm{r})$, bias correction factor $\left(C_{b}\right)$, concordance correlation coefficient $(\mathrm{CCC})$, location shift $(V)$, scale shift $(\mu)$, kappa $(\kappa) \pm \mathrm{SE}$, and maximum kappa $\left(\kappa_{\text {Max }}\right)$ values between the sensor and visual observations

\begin{tabular}{lcccccc}
\hline Item & $\mathrm{r}^{1}$ & $C_{b}$ & $\mathrm{CCC}^{2}$ & $V$ & $\mu$ & $\kappa\left(\kappa_{\text {Max }}\right)^{3}$ \\
\hline Behavior & & & & & & \\
$\quad$ Ruminating & 0.93 & 1.00 & 0.93 & -0.07 & 1.02 & $0.85 \pm 0.01(0.99)$ \\
Eating & 0.88 & 0.86 & 0.75 & -0.58 & 0.99 & $0.77 \pm 0.03(0.88)$ \\
Resting & 0.98 & 0.99 & 0.97 & -0.15 & 1.06 & $0.86 \pm 0.02(0.96)$ \\
Active & 0.73 & 0.49 & 0.35 & 1.26 & 0.49 & $0.47 \pm 0.03(0.77)$ \\
Overall & - & - & - & - & - & $0.78 \pm 0.01(0.93)$ \\
\hline
\end{tabular}

${ }^{1}$ The level of significance was for all behaviors $P<0.01$.

${ }^{2} \mathrm{CCC}=\mathrm{r} \times C_{b}$.

${ }^{3} \mathrm{Kappa}(\kappa)$ and $\kappa_{\operatorname{Max}}$ values as calculated based on Cohen (1960). The $\kappa$ value provides a measure for minuteby-minute agreement between observers, corrected for agreement that would be expected by chance. The $\kappa_{\text {Max }}$ indicates the maximum value of $\kappa$ that can be achieved when a difference exists between observers (or observer and sensor) in the number of minutes scored for each behavioral category.

was moderate ( $\kappa$ value of 0.47 ). For percentage of time agreement, the Pearson correlation and CCC between sensor and visual observation were high and low, respectively. The difference in absolute level of percentage of time active was reflected in a location shift of 1.26 . The scale shift of 0.49 indicates dissimilarity in the standard deviation of sensor and human estimated percentage of time active. Both location and scale shift resulted in a low CCC relative to the Pearson correlation.

The high agreement for the human versus human comparison for ruminating in the first experiment was in agreement with Schirmann et al. (2009) and Burfeind et al. (2011) (with heifers) and for rumination and eating with Rutter et al. (1997). The lower agreement in "active" was also seen in the lower concordance of "other behaviors" by Rutter et al. (1997). In conclusion, it can be stated that human observation is a reliable method to evaluate rumination, eating, and resting, with the overall category of "active" still classified well. However, differentiating between levels of activities is more difficult, as indicated by the low $\kappa$ values for the initial behavioral categories.

For the second experiment, correlation between sensor and human evaluation for ruminating expressed on total registration time was comparable with previous results of Schirmann et al. (2009), who used a neckcollar sensor based on sound recording, and expressed ruminating on the basis of 2-h registration blocks. Using 2-h blocks, the observed range in ruminating time is considerably higher than when using longer time blocks, due to a high within-day variation, resulting in a higher correlation between sensor and visual observation. For example, when the results in the current study are expressed in 2-h intervals, the correlation (r) between sensor and visual observation for ruminating improves from 0.93 to 0.97. Beauchemin et al. (1989) reported a very high $(>0.90)$ correlation between visual observation and automatic recording of behavior by making use of a stretch sensor in a halter around the mouth. In beef cattle, though, Goldhawk et al. (2013) reported correlations lower than 0.50 between rumination analyzed as minutes per 2-h intervals between a sensor and visual observation, using the same device as Schirmann et al. (2009).

Results for eating behavior and rumination were similar to those of Beauchemin et al. (1989), who also found lower correlation between visual and sensor observation for eating than for rumination. To the best of our knowledge, the present evaluation is the first one for a technology that monitors resting. Agreement for "active" was only moderate in the present study. However, positioning of these results in perspective with earlier literature is difficult because of different methodological approaches to defining active behavior. In the study of Roelofs et al. (2005) activity was monitored by counting steps, whereas Walker et al. (1996) made use of a pressure-sensing system to monitor mounting activity. Subsequently, both studies investigated whether an increasing level of activity, relative to a reference period, can be used as tool for estrus detection. The current study design was not suited for this purpose and, therefore, further research is recommended to evaluate the level of active behavior as defined by the current system in relation to estrus detection, should one aim to use the current system for this purpose.

Significant differences in absolute estimation of behavior between visual observation and sensor are not uncommon and have been reported as well by Kononoff et al. (2002) and Burfeind et al. (2011). Both of those studies indicated that sensor evaluation is likely to be more accurate. For the current study, it is difficult to speculate on the underlying causes for the absolute differences in estimates of percentage of behavior for "eating," "resting," and "active." The lower agreement between sensor and human observation for "active" is consistent with the results of the agreement between 
human observers for the initial categories (Table $1 ; \kappa$ 0.49 to 0.77 ). However, when these were condensed into 1 category of "active," agreement was almost perfect (Table $2 ; \kappa 0.96$ ). Ear movement patterns related to "eating" and "active" may be more complex and, thus, difficult to classify correctly, compared with a relatively simple and repetitive ear movement pattern for rumination.

In our view, practically, the sensor can be used to monitor ruminating and resting time in dairy cattle for both scientific purposes as well as for cow management. For "eating," the sensor is considered sufficiently reliable to be used for cow management. More research into the differences between human and sensor evaluation of activity could help in elucidating the causes for the differences found.

In conclusion, the results of this study provide strong evidence that the ear sensor technology described in this paper can be used to monitor ruminating and resting behavior in freestall-housed dairy cattle. Our results also suggest that this technology shows promise for monitoring eating behavior on commercial farms, but more work is needed to determine whether this technology can also be used for measuring activity of dairy cows.

\section{ACKNOWLEDGMENTS}

The authors thank the staff of Nutreco Ruminant Research Centre de Kempenshof (Boxmeer, the Netherlands). Furthermore, the authors thank Leo van Delft, Elshout, the Netherlands) for his contribution to the visual observations. Jasper Bikker performed this study as part of his MSc thesis in Animal Sciences at Wageningen University (Wageningen, the Netherlands), and was supervised by Jan Dijkstra, associate professor at Wageningen University. This project was the result of a research project between Nutreco R\&D and Agis Automatisering BV (Harmelen, the Netherlands).

\section{REFERENCES}

Beauchemin, K. A., S. Zelin, D. Genner, and J. G. Buchanan-Smith. 1989. An automatic system for quantification of eating and ruminating activities of dairy cattle housed in stalls. J. Dairy Sci. $72: 2746-2759$.

Burfeind, O., K. Schirmann, M. A. G. von Keyserlingk, D. M. Veira, D. M. Weary, and W. Heuwieser. 2011. Technical note: Evaluation of a system for monitoring rumination in heifers and calves. J. Dairy Sci. 94:426-430.

Cohen, J. 1960. A coefficient of agreement for nominal scales. Educ. Psychol. Meas. 20:37-46.

Ellis, J. L., A. Bannink, J. France, E. Kebreab, and J. Dijkstra. 2010. Evaluation of enteric methane prediction equations for dairy cows used in whole farm models. Glob. Change Biol. 16:3246-3256.

Friedman, H. 1982. Simplified determinations of statistical power magnitude of effect and research sample size. Educ. Psychol. Meas. 42:521-526.

Goldhawk, C., K. Schwartzkopf-Genswein, and K. A. Beauchemin. 2013. Technical note: Validation of rumination collars for beef cattle. J. Anim. Sci. 91:2858-2862.

Hinkle, D. E., W. Wiersma, and S. G. Jurs. 2003. Applied Statistics for the Behavioral Sciences. 5th ed. Houghton Mifflin, Boston, MA.

Kononoff, P. J., H. A. Lehman, and A. J. Heinrichs. 2002. Technical note: A comparison of methods used to measure eating and ruminating activity in confined dairy cattle. J. Dairy Sci. 85:18011803.

Landis, J. R., and G. G. Koch. 1977. The measurement of observer agreement for categorical data. Biometrics 33:159-174.

Lin, L. I. 1989. A concordance correlation coefficient to evaluate reproducibility. Biometrics 45:255-268.

Roelofs, J. B., F. J. van Eerdenburg, N. M. Soede, and B. Kemp. 2005. Pedometer readings for estrous detection and as predictor for time of ovulation in dairy cattle. Theriogenology 64:1690-1703.

Rutter, S. M., R. A. Champion, and P. D. Penning. 1997. An automatic system to record foraging behavior in free-ranging ruminants. Appl. Anim. Behav. Sci. 54:185-195.

Schirmann, K., M. A. G. von Keyserlingk, D. M. Weary, D. M. Veira, and W. Heuwieser. 2009. Technical note: Validation of a system for monitoring rumination in dairy cows. J. Dairy Sci. 92:6052-6055.

van Asseldonk, M. A. P. M., A. W. Jalvingh, R. B. M. Huirne, and A. A. Dijkhuizen. 1999. Potential economic benefits from changes in management via information technology applications on Dutch dairy farms: A simulation study. Livest. Prod. Sci. 60:33-44.

Walker, W. L., R. L. Nebel, and M. L. McGilliard. 1996. Time of ovulation relative to mounting activity in dairy cattle. J. Dairy Sci. 79:1555-1561. 